\title{
COULOMB ENERGIES OF SPHERICAL NUCLEI
}

\author{
JOACHIM JÄNECKE \\ Department of Physics, The University of Michigan, Ann Arbor, Michigan, USA ${ }^{\dagger}$
}

Received 5 July 1971

(Revised 21 October 1971)

\begin{abstract}
A bstract: A phenomenological Coulomb energy equation has been derived for spherical nuclei with diffuse surfaces. Contributions from the direct and exchange Coulomb energy and from the electromagnetic spin-orbit interaction are included explicitly. The experimental Coulomb displacement energies of 42 essentially spherical nuclei with $Z \geqq 28$ have been subjected to a least-squares analysis with the shape parameters of the ground state charge distributions required to agree with those obtained from electron scattering and muon capture X-ray experiments. Agreement between the experimental and calculated energies with a standard deviation of about $20 \mathrm{keV}$ is obtained if (i) the ratio between the rms radii of the neutron excess and the proton core is taken between about 1.10 and 1.15 , (ii) a smoothly $A$-dependent correction term which varies between about $+450 \mathrm{keV}$ in the light nuclei and $+350 \mathrm{keV}$ in the heavy nuclei is introduced, and (iii) an averaged contribution from the electromagnetic spin-orbit interaction is used rather than the expected shell-model contribution. Justification for this averaging may be provided by amounts of not less than $15 \%$ of excitations into higher shellmodel orbits for all nuclei. The Coulomb displacement energies of vibrational and rotational nuclei are observed to be smaller by up to $200 \mathrm{keV}$ than corresponding spherical nuclei. Neutron halos have been calculated and are found to be in very good agreement with the results of Hartree-Fock calculations, the droplet model of Myers and Swiatecki and the calculations by Nolen and Schiffer using wave functions generated in a Woods-Saxon potential well. The calculated neutron halo for ${ }^{208} \mathrm{~Pb}$ is $0.25 \pm 0.05 \mathrm{fm}$.
\end{abstract}

\section{Introduction}

Detailed Coulomb energy calculations which compare theory and experiment have been performed in the past ${ }^{1-11}$ ). Lists of earlier references are included in refs. ${ }^{1,12}$ ). Such calculations, however, are only for selected nuclei such as the mirror nuclei, the $\mathrm{Ca}, \mathrm{Ni}, \mathrm{Sn}$ isotopes and ${ }^{208} \mathrm{~Pb}$. Much more data exist, but only limited attention has been given to it. This is particularly true in the case of heavier nuclei with $Z \geqq 28$ for which the shell-model Coulomb energy equations of Carlson and Talmi ${ }^{13}$ ) or of Hecht ${ }^{14}$ ) are no longer applicable.

The present study was undertaken to derive a Coulomb energy equation for medium-heavy and heavy nuclei based upon more realistic density distributions for the proton core and the neutron excess. The purpose then was to subject the many data on Coulomb displacement energies to a least-squares analysis in order to relate the data to such quantities as the nuclear charge radius, the neutron halo, nuclear deformations, and their dependence on $A$. Another application is the prediction of nuclear masses, particularly for nuclei off the stability line.

t Work supported in part by the US Atomic Energy Commission. 
One of the problems which has to be solved is how to treat the correction terms. Presumably they include nuclear correlations, charge-dependent nuclear forces, Coulomb perturbations, nuclear rearrangements, and other effects ${ }^{1,5-11}$ ). They may add up to several hundred $\mathrm{keV}$. It appears that not all corrections are fully understood, a fact that may account for the so-called Coulomb energy anomaly ${ }^{1,2}$ ) which was originally considered to be a radius anomaly ${ }^{2}$ ). Nevertheless, considerable progress towards understanding these contributions has been made in recent years [refs. ${ }^{1,5-11}$ ] ] but quantitative agreement still appears to be lacking as has been demonstrated in the detailed calculations for the ${ }^{41} \mathrm{Sc}-{ }^{41} \mathrm{Ca}$ mirror nuclei. In the present paper no attempts are made at a detailed treatment of these correction terms. However, it was hoped that by including them as an adjustable quantity in an analysis of the data, one can perhaps obtain additional insight, e.g. on the dependence on $A$.

\section{Preliminary remarks}

Two-parameter Fermi distributions

$$
\rho(r)=\rho(0) f\left(\frac{r-c}{a}\right)=\frac{\rho(0)}{1+\exp \left(\frac{r-c}{a}\right)},
$$

are used throughout the present work to describe the radial matter density dependence of the protons and the neutron excess in the ground states of spherical nuclei. The quantity $c$ is the half-density radius and $a$ is the diffuseness. The skin thickness is $t \approx 4.4 a$.

The equivalent radius $R$ of any distribution is defined as the radius of a uniform density distribution with the same rms radius. Thus, $R=\sqrt{\frac{5}{3}}\left(\left\langle r^{2}\right\rangle^{\frac{1}{2}}\right.$. For a twoparameter Fermi distribution, the radii $c$ and $R$ are related according to

$$
\begin{aligned}
R^{2} & =c^{2}\left\{1+\frac{7}{3} \pi^{2}\left(\frac{a}{c}\right)^{2}\right\}=c^{2}+\frac{7}{3} \pi^{2} a^{2}, \\
R & =c\left\{1+\frac{7}{6} \pi^{2}\left(\frac{a}{c}\right)^{2}-\frac{49}{72} \pi^{4}\left(\frac{a}{c}\right)^{4}\right\}, \\
c & =R\left\{1-\frac{7}{6} \pi^{2}\left(\frac{a}{R}\right)^{2}-\frac{49}{72} \pi^{4}\left(\frac{a}{R}\right)^{4}\right\} .
\end{aligned}
$$

These equations are correct up to the fourth order in $(a / c)$ or $(a / R)$, respectively. Elton ${ }^{15,16}$ ) has shown that the half-density and equivalent radii of the nuclear charge distributions are quite well described by the equations

$$
\begin{aligned}
& R=r_{0} A^{\frac{1}{3}}\left\{1+\frac{5}{6} \pi^{2}\left(\frac{a}{r_{0} A^{\frac{1}{3}}}\right)^{2}-\frac{7}{24} \pi^{4}\left(\frac{a}{r_{0} A^{\frac{3}{3}}}\right)^{4}+\frac{1003}{1296} \pi^{6}\left(\frac{a}{r_{0} A^{\frac{1}{3}}}\right)^{6}\right\}, \\
& c=r_{0} A^{f}\left\{1 \frac{1}{3} \pi^{2}\left(\frac{a}{r_{0} A^{\frac{1}{5}}}\right)^{2}+\frac{1}{81} \pi^{6}\left(\frac{a}{r_{0} A^{\frac{1}{5}}}\right)^{6}\right\} .
\end{aligned}
$$


These equations were obtained by assuming both $a$ and $\rho(0)$ for the nuclear matter distribution to be independent of $A$. From electron scattering and muon capture $\mathrm{X}$-ray data Elton ${ }^{17}$ ) obtained $r_{0} \approx 1.135 \mathrm{fm}$ and $a \approx 0.513 \mathrm{fm}$ (average of the two sets of ref. ${ }^{17}$ ). Any Coulomb energy equation should therefore reproduce the experimental displacement energies with $R$ and $c$ from eqs. (6) and (7) and the above values for $r_{0}$ and $a$.

Fermi functions approximate the charge distribution of nuclear ground states quite well but not necessarily the distribution of the neutron excess, particularly when the neutron excess is small. However, Coulomb energies are very insensitive to the distribution of the charge in the interior of the nucleus and such an assumption introduces only small errors. This can easily be seen by assuming (for simplicity) a charge distribution of uniform density. The electric field $\varepsilon(r)$ is proportional to $r$ inside and proportional to $r^{-2}$ outside. The classical Coulomb energy

$$
E_{\mathrm{C}}=\frac{1}{2} e \int_{0}^{\infty} \rho(r) V(r) 4 \pi r^{2} \mathrm{~d} r=\frac{1}{8 \pi} \int_{0}^{\infty}[\varepsilon(r)]^{2} 4 \pi r^{2} \mathrm{~d} r,
$$

is obtained by integrating the function $\frac{1}{2} r^{2}[\varepsilon(r)]^{2}$ from zero to infinity. This function is proportional to $r^{4}$ inside and proportional to $r^{-2}$ outside the nuclear volume. The behavior of this function in conjuction with an extrapolation based on a more realistic distribution leads to the following general conclusions: (i) about $80 \%$ of the Coulomb encrgy results from the electric field outside the nucleus and is therefore independent of any internal structure, (ii) Coulomb energies depend strongly on the distribution of the charge near the surface (which is characterized by the radius and the diffuseness) and thus on the behavior of the wave functions near the surface, and (iii) Coulomb energies are insensitive to the behavior of the wave functions in the interior.

\section{The direct Coulomb energy $E_{\text {dir }}$}

The Fermi integral $F_{2}(k, x)$ is defined by

$$
F_{2}(k, x)=\int_{0}^{x} \xi^{2} f(\xi-k) \mathrm{d} \xi=\int_{0}^{x} \frac{\xi^{2} \mathrm{~d} \xi}{1+\exp (\xi-k)} .
$$

Using this integral, the total charge $Z e$, the electric field $\varepsilon(r)$, the electrostatic potential $V(r)$, and the direct Coulomb energy can be written as

$$
\begin{aligned}
& 7 e=e \rho(0) \int_{0}^{\infty} f\left(\frac{r^{\prime}-c}{a}\right) 4 \pi r^{\prime 2} \mathrm{~d} r^{\prime}=4 \pi a^{3} e \rho(0) F_{2}(c / a, \infty) .
\end{aligned}
$$

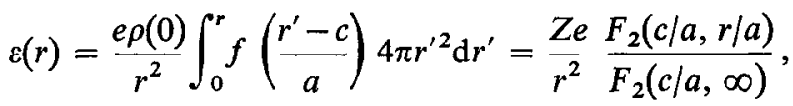

$$
\begin{aligned}
& V(r)=\int_{r}^{\infty} \varepsilon\left(r^{\prime}\right) \mathrm{d} r^{\prime}=\frac{Z e}{a F_{2}(c / a, \infty)} \int_{r / a}^{\infty} \frac{F_{2}(c / a, \xi)}{\xi^{2}} \mathrm{~d} \xi,
\end{aligned}
$$




$$
\begin{aligned}
E_{\mathrm{di} t} & =\left(1-\frac{1}{Z}\right) \int_{0}^{\infty} \frac{1}{2} e \rho\left(r^{\prime}\right) V\left(r^{\prime}\right) 4 \pi r^{\prime 2} \mathrm{~d} r^{\prime}=\left(1-\frac{1}{Z}\right) \frac{1}{8 \pi} \int_{0}^{\infty}\left[\varepsilon\left(r^{\prime}\right)\right]^{2} 4 \pi r^{\prime 2} \mathrm{~d} r^{\prime} \\
& =\frac{Z(Z-1) e^{2}}{c} \frac{(c / a)}{2\left[F_{2}(c / a, \infty)\right]^{2}} \int_{0}^{\infty} \frac{\left[F_{2}(c / a, \xi)\right]^{2}}{\xi^{2}} \mathrm{~d} \xi .
\end{aligned}
$$

The factor $(1-1 / Z)$ in eq. (13) has been introduced to remove the classical Coulomb self-energy. Thus, eq. (13) represents the quantum-mechanical direct Coulomb energy for a charge distribution $\rho(r)$ of $Z$ protons.

The value of the Fermi integral $F_{2}(k, x)$ is well known $\left.{ }^{18,19}\right)$ in the limit $x \rightarrow \infty$. It is equal to a polynomial in $k$ plus a correction term which is related to the integral for negative values of $k$. For finite values of $x$, an approximate expression can be obtained by expanding the function $f(r / a-c / a)$ as the sum of a step function and derivatives of $\delta$-functions ${ }^{19}$ ). The result is

$$
F_{2}(k, x) \approx \begin{cases}\frac{1}{3} x^{3} & \text { for } 0 \leqq x<k \\ \frac{1}{3} k^{3}+\frac{1}{3} \pi^{2} k & \text { for } k<x .\end{cases}
$$

This approximation, however, is generally not adequate, particularly for the direct Coulomb energy. As shown in detail in appendix $\mathrm{A}$, the function $F_{2}(k, x)$ can be represented exactly by a sum of three terms. The first and most important is that given by eq. (14). The second is an infinite sum resembling the Riemann $\zeta$-function but containing a polynomial in $x$ multiplied by powers of $e^{x-k}$ or $e^{k-x}$, respectively. The third term is a correction term identical to the one given by Rhodes ${ }^{18}$ ) for the integral $F_{2}(k, \infty)$.

The necessity for an exact representation of $F_{2}(k, x)$ is demonstrated in fig. 1 which shows the radial dependence of the charge $\rho(r)$ and the electric field $\varepsilon(r)$ for the case $c / a=10$. The $\delta$-function approximation for the Fcrmi function gencrates a spike at $r=c$ in the radial dependence of $\varepsilon(r)$. The exact expression gives the expected smooth behavior. Also shown in fig. 1 (dotted lines) are the fields $\varepsilon(r)$ corresponding to two uniform density distributions for which the half-density radius $c$ and the equivalent radius $R$ are equal to $c$ and $R$ of the diffuse surface distribution, respectively. These ficlds $\varepsilon(r)$ have little resemblance to the true field (except, of course, outside the charge distribution).

The direct Coulomb energy $E_{\text {dir }}$ can be calculated to any desired accuracy using eq. (13) coupled with the exact representation of $F_{2}(c / a, \xi)$ as given in appendix $A$. The procedure consists of expanding the integral of eq. (13) in decreasing powers of $c / a$ and replacing $c$ by $R$ according to eq. (5). The result is

$$
E_{\mathrm{dir}}=\frac{3}{5} \frac{Z(Z-1) e^{2}}{R} C_{\mathrm{dir}}
$$

with

$$
C_{\mathrm{dir}}=1+b_{1}\left(\frac{a}{R}\right)^{3}+b_{2}\left(\frac{a}{R}\right)^{4}
$$


Here, $b_{1}=18.0295$ [derived earlier by Lindner $\left.{ }^{20}\right)$ ] and $b_{2}=-85.2330$. The detailed expressions for $b_{1}$ and $b_{2}$ (see appendix B) contain the Riemann $\zeta$-function as well as other infinite series. The correction term $C_{\mathrm{dir}}$ and all other correction terms to be derived are through fourth order in $(a / R)$.

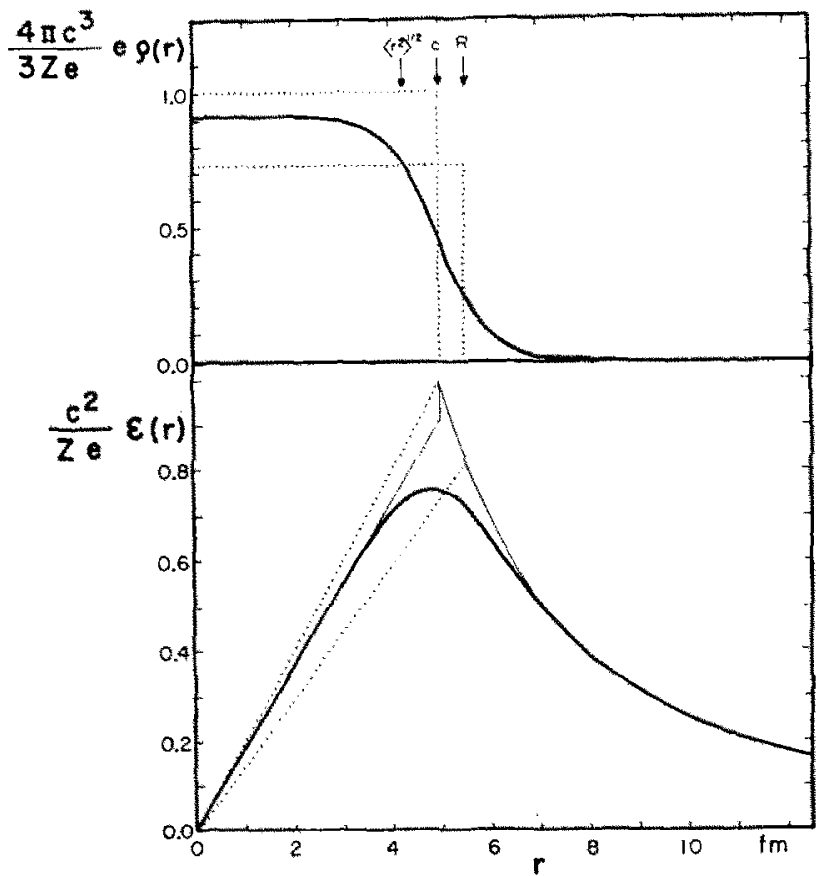

Fig. 1. Charge density $e \rho(r)$ and electric field $\varepsilon(r)$ for a distribution of Fermi shape with $c=5 \mathrm{fm}$ and $a=0.5 \mathrm{fm}$ (heavy lines). The thin line for $\varepsilon(r)$ represent the results based on the $\delta$-function approximation for $f((r-c) / a)$ (upward spike). The dotted lines for $e \rho(r)$ and $\varepsilon(r)$ represent the results for uniform density distributions which have the same values for the half-density radius $c$ or the equivalent radius $R$, respectively. Note the change in density in the interior.

It is interesting to note that the first nonvanishing correction term in $C_{\mathrm{dir}}$ is of third order in $a / R$. This result explains why eq. $(15)$ with $C_{\text {dir }} \approx 1$, which has often been used in the past, is a good approximation for $E_{\text {dir }}$. Eq. (15) will not be used below to calculate the energy difference $\Delta E_{\mathrm{dir}}$ between analogue states. Instead, a more elaborate procedure presented in sect. 4 will be followed.

\section{The direct Coulomb energy difference $\Delta E_{\text {dir }}$}

The displacement energy between neighboring analogue states can be written as

$$
\Delta E=\Delta E_{\mathrm{dir}}(1-\varepsilon)+\Delta E_{\text {s.o. }}+\Delta E_{\text {corr }} .
$$

The quantity $\Delta E_{\mathrm{dir}}$ represents the difference in the direct Coulomb energy. The quantity $\varepsilon$ is the so-called exchange factor. It is defined as the ratio $-\Delta E_{\text {exch }} / \Delta E_{\mathrm{dir}}$, where 
$\Delta E_{\text {exch }}$ is the Coulomb energy due to the antisymmetrization of the many-particle wave function. The quantity $\Delta E_{\text {s.o. }}$ is the difference in the contributions from the electromagnetic spin-orbit interaction. The quantity $\Delta E_{\text {corr }}$ is a correction term which results from numerous small and not-so-small effects such as Coulomb perturbations to the radial wave function of the excess proton, nuclear rearrangements of the core due to the presence of an additional charge, isospin impurities of the core, nuclear correlations, the dynamic proton-neutron mass effect, finite size corrections, chargedependent nuclear forces and many others. These effects have been discussed in detail by other authors ${ }^{1, s-11}$ ).

The difference in the direct Coulomb energy will be calculated on the assumption that there exists no isospin impurities in the core and that the analogue state wave functions are obtained from that of the parent state (ground state) by applying the $T$-lowering operator. Thus, the shape of the wave functions of the excess neutrons in the ground state and of the excess proton in the analogue state is assumed to be identical. Such an assumption is, of course, only approximately valid because of effects such as the Coulomb perturbations of the wave function for the excess proton and the polarization of the core due to the replacement of a neutron by a proton. Any energy shifts from these effects are assumed to be included in the correction term $\Delta E_{\text {corr }}$.

Based on the above assumption, the direct Coulomb energy difference can be written as

$$
\Delta E_{\mathrm{dir}}=\frac{e}{N-Z} \int_{0}^{\infty} \rho_{\mathrm{exc}}\left(r^{\prime}\right) V_{\mathrm{core}}\left(r^{\prime}\right) 4 \pi r^{\prime 2} \mathrm{~d} r^{\prime},
$$

where $\rho_{\text {exc }}(r)$ is the density distribution of the $(N-Z)$ excess neutrons in the ground state of the parent nucleus and $V_{\text {core }}(r)$ is the electrostatic potential generated by the $Z$ protons of the core. As pointed out before, the densities $\rho_{\text {core }}(r)$ and $\rho_{\text {exc }}(r)$ will be represented by Fermi functions characterized by $c, a$ and $c^{\prime}, a^{\prime}$, respectively. When the respective replacements are made, one obtains after one partial integration

$$
\Delta E_{\mathrm{dir}}=\frac{Z e^{2}}{c} \frac{(c / a)}{\left(a^{\prime} / a\right)^{2} F_{2}(c / a, \infty) F_{2}\left(c^{\prime} / a^{\prime}, \infty\right)} \int_{0}^{\infty} \frac{F_{2}(c / a, \xi) F_{2}\left(c^{\prime} / a^{\prime},\left(a / a^{\prime}\right) \xi\right)}{\xi^{2}} \mathrm{~d} \xi,
$$

where $F_{2}(k, x)$ is the Fermi integral discussed in appendix A. Eq. (19) corresponds to eq. (13) if $a^{\prime}=a$ and $c^{\prime}=c$. The evaluation of the integral in eq. (19) is straightforward but tedious. Here, we will present only the results for the somewhat simplified case with $a^{\prime}=a$. The difficulty in evaluating the above integral stems from the fact that three regions have to be considered. The regions are from 0 to $c / a$ to $c^{\prime} / a$ to infinity. It will be assumed that $c^{\prime}>c$. The integral can be expanded in decreasing powers of $c / a$ (and $c^{\prime} / a$ ), but unlike the integral of eq. (13), the coefficients of the various terms in the expression are not constants. They are functions of $c / a$ and $h \equiv c^{\prime} / c$. The result (for details see appendix B) is

$$
\Delta E_{\mathrm{dir}}=\frac{6}{5} \frac{Z e^{2}}{c} \frac{b_{3}(h)+b_{4}(h, c / a)(a / c)^{2}+b_{5}(h, c / a)(a / c)^{3}+b_{6}(h, c / a)(a / c)^{4}}{\left[1+\pi^{2}(a / c)^{2}\right]\left[1+\pi^{2}(a / h c)^{2}\right]}
$$


with

$$
\begin{aligned}
b_{3}(h) & =\frac{5 h^{2}-1}{4 h^{3}}, \\
b_{4}\left(h, \frac{c}{a}\right) & =\frac{5 \pi^{2}\left(3 h^{2}-1\right)}{12 h^{3}}+\frac{5\left(h^{3}-1\right)}{2 h^{3}} \zeta\left(2,(h-1) \frac{c}{a}\right), \\
b_{5}\left(h, \frac{c}{a}\right) & =\frac{1+h^{2}}{2 h^{3}}\left[b_{1}-15 \zeta(3)+15 \zeta\left(3,(h-1) \frac{c}{a}\right)\right], \\
b_{6}\left(h, \frac{c}{a}\right) & =-\frac{\pi^{4}}{6 h^{3}}+\frac{5(h-1)}{2 h^{3}} \zeta\left(2,(h-1) \frac{c}{a}\right) .
\end{aligned}
$$

The quantity $b_{1}$ is that of eq. (16). The functions $\zeta(2, x)$ and $\zeta(3, x)$ are defined by

$$
\zeta(n, x) \equiv \sum_{l=1}^{\infty} \frac{\mathrm{e}^{-l x}}{l^{n}} .
$$

We have $\zeta(n, 0)=\zeta(n)$ which is the Riemann $\zeta$-function.

The half-density radius $c$ of the proton core and the ratio $h \equiv c^{\prime} / c$ are related to the equivalent radius $R$ and the ratio $f \equiv\left\langle r_{\text {exc }}^{2}\right\rangle^{\frac{1}{2}} /\left(r_{\text {core }}^{2}\right\rangle^{\frac{1}{2}}=R^{\prime} / R$ according to eq. (5) and

$$
h=\sqrt{f^{2}+\left(f^{2}-1\right) \frac{7}{3} \pi^{2}(a / c)^{2}},
$$

respectively. Eq. (26) can be verified by making use of eq. (3). It is not practical, however, to explicitly replace $c$ and $h$ in eq. (20) by $R$ and $f$.

In the limit $f \rightarrow 1$ (or $h \rightarrow 1$ ) we obtain $b_{3}(h) \rightarrow 1, b_{4}(h, c / a) \rightarrow \frac{5}{6} \pi^{2}, b_{5}(h, c / a) \rightarrow b_{1}$, $b_{6}(h, c / a) \rightarrow \frac{1}{4} \pi^{4}$. If one now replaces $c$ by $R$, eq. (20) reduces to

$$
\Delta E_{\mathrm{dir}} \approx \frac{6}{5} \frac{Z e^{2}}{R} C_{\mathrm{dir}}
$$

with $C_{\mathrm{dir}}$ from eq. (16). The approximate eq. (27) can, of course, be derived directly from eq. (15). It confirms that the diffuseness correction in eq. (20) is relatively weak.

The leading term in the dependence of $\Delta E_{\mathrm{dir}}$ on the ratio $f=R^{\prime} / R$ can perhaps best be recognized, in the limit $a \rightarrow 0$. The result is

$$
\Delta E_{\mathrm{dir}} \approx \frac{6}{5} \frac{Z e^{2}}{R} \frac{5 f^{2}-1}{4 f^{3}} .
$$

Eq. (28) is not valid for $f<1$ or $R^{\prime}<R$. The correct equation for that case can be obtained by the replacement $R \leftrightarrow R^{\prime}$. Eq. (28) has an important consequence. If $R$ or $R^{\prime}$ are kept constant and the respective other radius is varied, one obtains

$$
\begin{aligned}
& \frac{\delta \Delta E_{\mathrm{dir}}}{\Delta E_{\mathrm{dir}}} \approx-\frac{5 f^{2}-3}{5 f^{2}-1} \frac{\delta R^{\prime}}{R^{\prime}} \quad \text { if } \quad R=\text { const., } \\
& \frac{\delta \Delta E_{\mathrm{dir}}}{\Delta E_{\mathrm{dir}}} \approx-\begin{array}{c}
\delta R \\
5 f^{2}-1
\end{array} \quad \text { if } \quad R^{\prime}=\text { const. }
\end{aligned}
$$


Both equations combined give

$$
\frac{\delta R}{R}+\alpha \frac{\delta R^{\prime}}{R^{\prime}} \approx 0 \quad \text { if } \Delta E_{\mathrm{dir}}=\text { const., }
$$

or

$$
R R^{\prime a} \approx \text { const. } \quad \text { if } \Delta E_{\mathrm{dir}}=\text { const. }
$$

Here, $\alpha=\frac{1}{2}\left(5 f^{2}-3\right)$. Eqs. (31) and (32) show that for $f$ in the range from 1.10 to 1.15 the direct Coulomb energy difference $\Delta E_{\mathrm{dir}}$ is 1.5 to 1.8 times as sensitive to a change in $R^{\prime}$ than it is to a change in $R$. For $f \approx 1$ the dependence on $R$ and $R^{\prime}$ is about the same ${ }^{1}$ ).

Eqs. (29)-(32) also show that it is impossible to search independently for the radii of the core and the neutron excess. Not unless information exists (or assumptions are made) about the radius of the neutron excess can charge radii be determined from experimental Coulomb displacement energies or not unless the charge radii are known can the radius of the neutron excess or the neutron halo be determined.

\section{The exchange factor}

Wave functions for many-nucleon systems have to be antisymmetrized. It is for this reason that the (negative) Coulomb exchange energy has to be added to the previously calculated direct Coulomb energy. Eq. (17) requires an evaluation of the so-called exchange factor $\varepsilon=-\Delta E_{\text {exch }} / \Delta E_{\mathrm{dir}}$.

Based on the statistical Fermi-gas model for the nucleus, Bethe and Bacher ${ }^{21,22}$ ) derived the expression

$$
E_{\mathrm{exch}}=-\frac{3}{2}\left(\frac{3}{8 \pi}\right)^{\frac{1}{t}} e^{2} \int_{0}^{\infty}[\rho(r)]^{4 / 3} 4 \pi r^{2} \mathrm{~d} r
$$

As pointed out by Carlson and Talmi ${ }^{13}$ ) (see also refs ${ }^{23,1}$ )), this expression includes the classical Coulomb self-energy. To compensate this contribution, the equation

$$
E_{\mathrm{exch}}=\tilde{E}_{\mathrm{exch}}+\frac{1}{Z} E_{\mathrm{dir}},
$$

will be used instead of eq. (33).

With the use of eqs. (2) and (10) the eq. (33) for $E_{\text {exeh }}$ can be rewritten as

$$
\widetilde{E}_{\text {exch }}=-\frac{e^{2} Z^{4 / 3}}{a}\left(\frac{9}{16 \pi}\right)^{\xi} \frac{\int_{0}^{\infty} \xi^{2}[f(\xi-c / a)]^{4 / 3} \mathrm{~d} \xi}{\left[F_{2}(c / a, \infty)\right]^{4 / 3}} .
$$

The integral in eq. (35) cannot be reduced to any of the Fermi integrals discussed in appendix A. However, the evaluation is very similar if instead of the power series expansions (A.9) for the function $f(\xi-k)$ the corresponding expansions for the function $[f(\xi-k)]^{*}$ is used (see appendix B). One again has to integrate by parts and 
replace $c$ by $R$. The result is

$$
\tilde{E}_{\mathrm{exch}}=-3\left(\frac{3}{16 \pi}\right)^{\frac{2}{3}} \frac{e^{2} Z^{4 / 3}}{R} C_{\text {exch }} .
$$

Here

$$
C_{\text {exch }}=1+b_{7}\left(\frac{a}{R}\right)+b_{8}\left(\frac{a}{R}\right)^{2}+b_{9}\left(\frac{a}{R}\right)^{3}+b_{10}\left(\frac{a}{R}\right)^{4}
$$

is a correction term and $b_{7}=-1.3356, b_{8}=7.1270, b_{9}=-18.2104$ and $b_{10}=$ 83.4059. The above expression is well known for $a=0$ when $C_{\text {exch }}=1$. The interesting fact, however, is that the correction to the exchange energy is of the first order in $(a / R)$ and therefore appreciable.

The next step is to calculate the exchange factor $\varepsilon$. Since $\varepsilon$ is a correction of only a few percent, one is justified in using for $\Delta E_{\text {exch }}$ and $\Delta E_{\mathrm{dir}}$ the approximation which assumes the ground state and analogue state charge radii to be equal $(f=h=1)$. Thus

$$
\begin{aligned}
\Delta E_{\mathrm{exch}} & =-4\left(\frac{3}{16 \pi}\right)^{\frac{2}{3}} \frac{e^{2}\left(Z+\frac{1}{2}\right)^{\frac{3}{3}}}{R} C_{\mathrm{exch}}+\frac{3}{5} \frac{e^{2}}{R} C_{\mathrm{dir}}, \\
\varepsilon & =\frac{10}{3}\left(\frac{3}{16 \pi}\right)^{\frac{2}{3}} \frac{\left(Z+\frac{1}{2}\right)^{\frac{1}{3}}}{Z} \frac{C_{\mathrm{exch}}}{C_{\mathrm{dir}}}-\frac{1}{2 Z} .
\end{aligned}
$$

Nolen and Schiffer ${ }^{1}$ ) compared exchange factors calculated for different nuclear models. Table 1 shows their results based on the oscillator, the Woods-Saxon and on

TABLE 1

Exchange factors $\varepsilon$ in $\%$ calculated by Nolen and Schiffer ${ }^{1}$ ) for the oscillator, the Woods-Saxon and the statistical models and comparison with the results of eq. (39) where the diffuseness of the nuclear surface is taken into account

\begin{tabular}{rccccc}
\hline$Z<$ & $A$ & $\begin{array}{c}\text { Oscillator } \\
\text { model }\end{array}$ & $\begin{array}{c}\text { Woods-Saxon } \\
\text { model }\end{array}$ & $\begin{array}{c}\text { Statistical } \\
\text { model }\end{array}$ & $\begin{array}{c}\text { Statistical-model } \\
\text { with diffuseness }\end{array}$ \\
\hline 6 & 13 & 7.1 & 6.7 & 11.8 & 6.1 \\
8 & 17 & 5.6 & 5.4 & 9.7 & 5.6 \\
14 & 29 & 4.1 & 3.8 & 7.0 & 4.5 \\
16 & 33 & 4.3 & 4.4 & 6.5 & 4.3 \\
20 & 41 & 3.7 & 3.7 & 5.7 & 3.9 \\
28 & 62 & & 3.2 & 4.6 & 3.3 \\
50 & 120 & & 2.2 & 3.3 & 2.5 \\
82 & 208 & & 1.8 & 2.4 & 1.9 \\
\hline
\end{tabular}

the statistical models. The exchange factors from the statistical Fermi-gas model are systematically higher by about $80 \%$ in the light and by about $40 \%$ in the heavy nuclei than those obtained using wave functions generated in the potential wells. The last column in table 1 , however, shows that the discrepancy is completely removed when the diffuseness of the nuclear surface is taken into consideration as prescribed by eq. 
(39). The agreement includes the light nuclei which is surprising in view of the fact that eq. (33) is based on a statistical model.

\section{The electromagnetic spin-orbit energy difference $\Delta E_{\text {s.o. }}$}

The only other correction term which will be calculated explicitly is that due to the electromagnetic spin-orbit interaction. The expression for the spin-orbit energy for atomic electrons is well known ${ }^{24}$ ). When applied to the neutrons and protons in a nucleus which has a spherical electrostatic potential $V_{\text {cere }}$, the result is

$$
E_{\text {s.o. }}=\sum_{i=1}^{A}\left\langle\frac{e \hbar^{2}}{2 m_{\mathrm{p}}^{2} c_{l}^{2}} \frac{1}{r} \frac{\mathrm{d} V_{\text {core }}}{\mathrm{d} r}\left[\frac{1}{2}\left(g_{\mathrm{n}}+g_{\mathrm{p}}-1\right)+\left(g_{\mathrm{n}}-g_{\mathrm{p}}+1\right) t_{z i}\right] \boldsymbol{t}_{i} \cdot s_{i}\right\rangle .
$$

Note that the Thomas precession requires the $g$-factors to be replaced by $g-q /|q|$; thus $g_{\mathrm{n}}$ and $g_{\mathrm{p}}-1$ are entered into eq. (40).

The difference between the electromagnetic spin-orbit interaction for the analogue state and the ground state is

$$
\Delta E_{\mathrm{s} . e .}=-\frac{1}{N-Z} \sum_{\text {excess }}\left\langle\frac{e \hbar^{2}}{2 m_{\mathrm{p}}^{2} c_{t}^{2}} \frac{1}{r} \frac{\mathrm{d} V_{\text {core }}}{\mathrm{d} r}\left(g_{\mathrm{n}}-g_{\mathrm{p}}+1\right) l \cdot s\right\rangle,
$$

which reduces to

$$
\Delta E_{\text {s.o. }}=\left(g_{\mathrm{p}}-g_{\mathrm{n}}-1\right) \frac{e \hbar^{2}}{2 m_{\mathrm{p}}^{2} c_{l}^{2}}\left\{\frac{1}{N-Z} \sum_{\text {excess }} l \cdot s\right\}\left\{\frac{1}{N-Z} \int_{0}^{\infty} \rho_{\text {exc }}(r) \frac{1}{r} \frac{\mathrm{d} V_{\text {core }}}{\mathrm{d} r} 4 \pi r^{2} \mathrm{~d} r\right\} \text {. }
$$

When Fermi functions are introduced, eq. (42) can be rewritten as

$$
\begin{aligned}
\Delta E_{\mathrm{s.o.} .}=-\left(g_{\mathrm{p}}-g_{\mathrm{n}}-1\right)\left(\frac{e h}{2 m_{\mathrm{p}} c_{\mathrm{l}}}\right)^{2}\left\{\frac{1}{N-Z} \sum_{\mathrm{excess}} l \cdot \sigma\right\} \frac{Z}{c^{3}} \\
\times\left\{\left(\frac{c}{a}\right)^{3} \frac{\left.\int_{0}^{\infty} f(\xi-h c / a) \frac{1}{\xi} F_{2}(c / a, \xi) \mathrm{d} \xi\right\}}{F_{2}(c / a, \infty) F_{2}(h c / a, \infty)}\right\} .
\end{aligned}
$$

Since $\Delta E_{\text {s.o. }}$ is only a relatively small correction term, the integral in eq. (43) can be evaluated in the $\delta$-function approximation of eq. (A.14). The result is (see appendix B)

$$
\Delta E_{\text {s.o. }}=-\left(g_{\mathrm{p}}-g_{\mathrm{n}}-1\right) \mu_{0}^{2}\left\{\frac{1}{N-Z} \sum_{\text {excess }} l \cdot \sigma\right\} \frac{Z}{c^{3}} C_{\text {s.o. }},
$$

with the correction term

$$
C_{\text {s.o. }}=\frac{(1+3 \ln h)+\pi^{2}\left(3 \ln h-1 / 2 h^{2}\right)(a / c)^{2}-\left(\pi^{4} / 2 h^{2}\right)(a / c)^{4}}{h^{3}\left[1+\pi^{2}(a / c)^{2}\right]\left[1+\pi^{2}(a / h c)^{2}\right]} .
$$

Here, $\mu_{0}$ is the nuclear magneton and $\left(g_{p}-g_{\mathrm{n}}-1\right) \mu_{0}^{2}=134 \mathrm{keV} \cdot \mathrm{fm}^{3}$. The halfdensity radius $c$ and the ratio $h=c^{\prime} / c$ have not been explicitly replaced by $R$ and $f=$ 
$R^{\prime} / R$. To do so, eqs. (5) and (26) have to be used. Such replacements are only practical if further approximations are made [see eqs. (61) and (65)]. Numerical values for $\Delta E_{\text {s.o. }}$ extracted from eq. (44) agree to within a few $\mathrm{keV}$ with results obtained by using wave functions generated in a Woods-Saxon wall ${ }^{1,4,5}$ ).

A problem arises in the numerical evaluation of the sum $\sum_{\text {excess }} l \cdot \sigma$. Only if nuclear configurations are known or are assumed to be known can the sum be calculated. If a great number of nuclei is treated simultaneously, as for example is the case in a leastsquares analysis, one needs a general prescription for the determination of the sum.

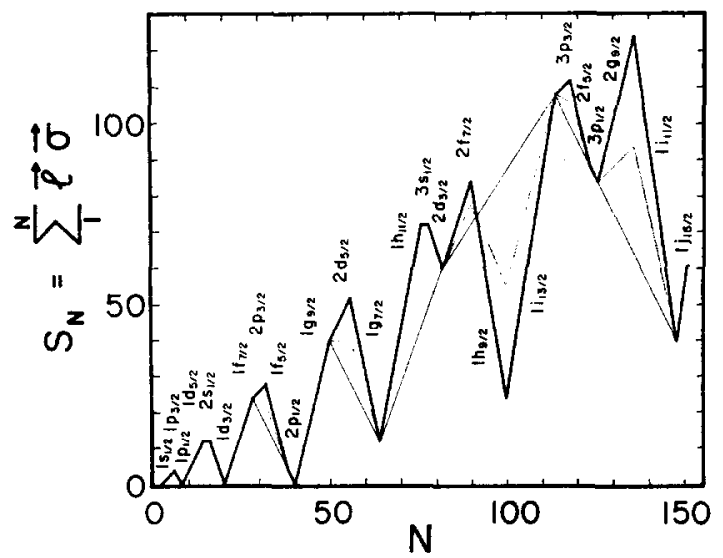

Fig. 2. Plot of the spin-orbit sum $S_{N}=\Sigma_{1}^{N} I \cdot \sigma$ as a function of $N$. The heavy, thin and dotted lines were obtained assuming an extreme single-particle shell model, complete subshell mixing, and an intermediate scheme (see text).

Fig. 2 shows the sum $S_{N}=\sum_{1}^{N} \boldsymbol{l} \cdot \boldsymbol{\sigma}$ derived under several assumptions. The sum over the excess neutrons is then obtained from $S_{N}-S_{z}$. The heavy solid line is obtained by assuming an extreme single-particle shell model with a sequence of orbits as indicated. The sum $S_{N}$ is a gradually increasing function of $N$ due to the shell-model spin-orbit splitting. A positive contribution from the neutrons in the $1 \mathrm{~g}_{\frac{2}{2}}$ orbit, for example, is completely compensated by those in the $\lg _{\frac{7}{2}}$ orbit, but only at a higher neutron number. The thin solid line is obtained by introducing complete mixing between some of the subshells such as the $2 \mathrm{p}_{\frac{3}{2}}, 1 \mathrm{f}_{\frac{3}{2}}, 2 \mathrm{p}_{\frac{1}{2}}$, the $2 \mathrm{~d}_{\frac{3}{2}}, 1 \mathrm{~g}_{\frac{7}{2}}$, the $1 \mathrm{~h}_{\frac{11}{2}}, 3 \mathrm{~s}_{\frac{1}{2}}, 2 \mathrm{~d}_{\frac{3}{2}}$, orbits, etc. The dotted line, finally, is obtained essentially as the average between the two beforementioned procedures. It is presumably the most realistic assumption and has been used in the least-squares analysis discussed in sect. 7. In a number of cases values for $S_{N}$ from fig. 2 were compared to values for $S_{N}$ calculated from known wave functions [refs. $\left.{ }^{25,26}\right)$ ], and the agreement was quite satisfactory.

\section{Least squares analysis of the experimental Coulomb displacement energies}

One of the major objectives of the present work was to subject the many data on Coulomb displacement energies to a least-squares analysis. Since correlations exist 
between the various quantities of interest, care must be taken in deciding which ones to use as adjustable parameters. In the least-squares analysis (the results of which are presented below), the radius and diffuseness parameters for the charge distribution of the core were required to agree with those derived from electron scattering and muonic X-ray experiments. Distributions of Fermi shape were assumed and eqs. (6) and (7) were used to describe the $A$-dependence of the equivalent radius $R$ and the half-density radius $c$. The parameters $r_{0}$ and $a$ were taken as $r_{0}=1.135 \mathrm{fm}$ and $a=0.513 \mathrm{fm}$ [ref. $\left.\left.{ }^{17}\right)\right]$.

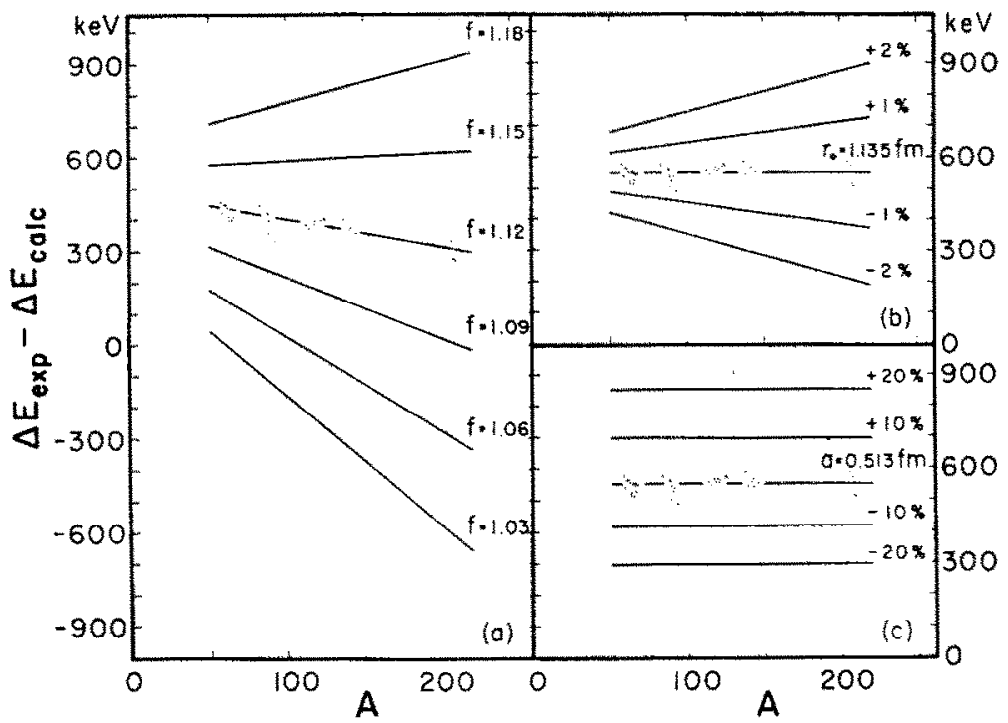

Fig. 3. Difterence between the experimental and calculated Coulomb displacement energies as a function of $A$ with (a) variable ratio $f \equiv R^{\prime} / R$ and $r_{0}=1.135 \mathrm{fm}, a=0.513 \mathrm{fm}, \Delta E_{\text {corr }}=0 \mathrm{keV}$; (b) variable radius parameter $r_{0}$ and $f=1.144, a=0.513 \mathrm{fm}, \Delta E_{\text {corr }}=0 \mathrm{keV}$; (c) variable diffuseness $a$ and $r_{a}=1.135 \mathrm{fm}, f=1.144, \Delta E_{\text {corr }}=0 \mathrm{keV}$.

First consider the various correlations including those involving $r_{0}$ and $a$. Fig. 3 shows the dependence of the difference between the experimental and calculated displacement energies as a function of $A$ for a variety of parameters. To achieve clarity, the individual points are shown only in a few cases while straight lines represent the general relationships. In each of the sets (a), (b) and (c) all but one of the parameters were kept constant. The experimental Coulomb displacement energies were taken from ref. ${ }^{12}$ ). Only data for $Z \geqq 28$ were used. Since it was soon discovered that the experimental energies of nuclei with statically or dynamically deformed shapes are systematically low by up to $200 \mathrm{keV}$, these nuclei were excluded at this stage. Only nuclei which are semi-magic or nearly semi-magic were included, namely those with $Z=Z_{0}, Z_{0} \pm 1$ or $N=N_{0}, N_{0} \pm 1$. These nuclei are presumably nearly spherical in shape. 
Fig. 3 can be interpreted in two ways. The ordinate may represent the deviation of the experimental from the calculated values for the given parameters or it may represent the correction term $\Delta E_{\text {corr }}$ which is required to make $\Delta E_{\text {calc }}$ to agree with $\Delta E_{\text {exp }}$. The lines shown on the figures reflect the correlations which exist between the parameters. Empirically, these are

$$
\Delta E_{\text {corr }} \approx[36(f-1.144)(A+79)+550],
$$

if $r_{0}=1.135 \mathrm{fm}$ and $a=0.513 \mathrm{fm}$,

$$
\begin{gathered}
1.35 \frac{\Delta r_{0}}{r_{0}}+\frac{\Delta f}{f} \approx 0, \\
\frac{\hat{o} \Delta E_{\text {corr }}}{\partial a} \approx+2730 \frac{\mathrm{keV}}{\mathrm{fm}} \quad(\approx \text { independent of } A) .
\end{gathered}
$$

Eq. (47) agrees with the earlier prediction of eqs. (31) and (32). Since $\Delta E_{\text {calc }}$ decreases with increasing diffuseness $a$, the correction term $\Delta E_{\text {corr }}$ has to be increased accordingly. The dependence on $a$ according to eq. (48) is quite strong. However, this is in part due to the fact that a variation in the diffuseness $a$ with constant $r_{0}$ will also change both $R$ and $c$. The density in the nuclear interior, on the other hand, remains constant.

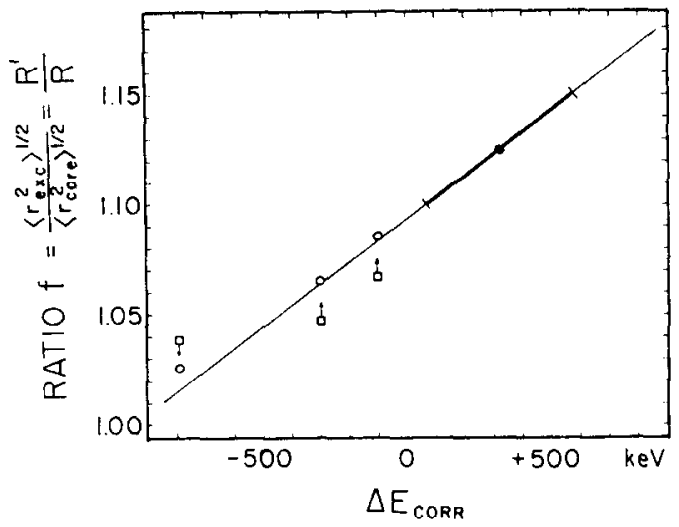

Fig. 4. Calculated correlation between the ratio $f \equiv R^{\prime} / R$ and $\Delta E_{\text {corr }}$ for $Z=82, A=208$. The line represents those values of $f$ which are required to reproduce the experimental Coulomb displacement energy of ${ }^{208} \mathrm{Bi}-{ }^{208} \mathrm{~Pb}$ for a given correction $\Delta E_{\text {corr }}$. The filled circle with the error bar results from the present analysis; the open squares are from the literature ${ }^{4,5}$ ); the open circles make use of an adjusted value for the charge radius (see text).

If $r_{0}$ and $a$ are fixed at $r_{0}=1.135 \mathrm{fm}$ and $a=0.513 \mathrm{fm}$ to agree with the results ${ }^{17}$ ) of electron scattering and muon capture X-ray experiments, only eq. (46) is of significance. Thus, the correction term $\Delta E_{\text {corr }}$ and the ratio $f \equiv R^{\prime} / R$ remain strongly correlated. As a special example, fig. 4 shows the calculated dependence of $f$ on the magnitude of the correction term $\Delta E_{\text {orr }}$ for ${ }^{208} \mathrm{~Pb}$. The results of the analyses by Friedman and Mandelbaum ${ }^{4}$ ), Auerbach et al. ${ }^{5}$ ) are shown as open squares (addi- 
tional values including $f=1.08$ were reported by Nolen and Schiffer $\left.{ }^{1}\right)$ ). Corrected values are represented by open circles. They were obtained from those represented by open squares by using $R$ from eq. (6) and requiring $R R^{\prime} \approx$ constant. The fact that the open circles are very close to the calculated line shows that the equations presented in this paper do indeed give results which agree well with those derived from realistic wave functions. The filled circle with the error bar represents the best estimate for $f$ based on the subsequent least-squares analysis.

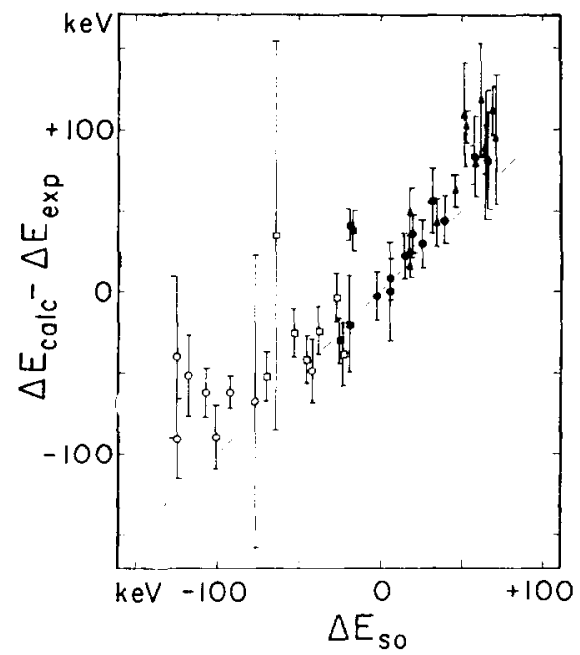

Fig. 5. Plot of the difference between the calculated and experimental Coulomb displacement energies as a function of the electromagnetic spin-orbit interaction correction term $\Delta E_{\text {s.o. }}$. The results shown are for $r_{0}=1.135 \mathrm{fm}, a=0.513 \mathrm{fm}, f=1.144, \Delta E_{\text {corr }}=550 \mathrm{keV}$. Only semimagic nuclei and nearly semi-magic nuclei have been included. The filled and open triangles, circles and squares denote: $\triangle Z=28,29 ; \bigcirc N=49,50,51 ; \bigcirc Z=49,50,51 ; \square N=81,82,83 ; \square Z=81$, 82,83 .

Special assumptions have to be introduced to make a least-squares analysis possible. One possibility is to assume that both $\Delta E_{\text {corr }}$ and $f$ are independent of $A$. Other assumptions, however, are also possible and will be discussed later.

The weight factors for the individual energies were calculated by quadratically adding a fixed energy $\delta \Delta E$ to the experimental uncertainties. Thus, $p_{i}=1 /\left(\delta \Delta E_{i}^{2}+\right.$ $\left.\delta \Delta E^{2}\right)$. A value of $\delta \Delta E=20 \mathrm{keV}$ was considered reasonable. In doing so, experimental values with quoted uncertainties much less than $20 \mathrm{keV}$ are not given excessive weight factors. The values for $\chi^{2}$ which are obtained by this procedure are reduced and have to be reinterpreted ${ }^{27}$ ). The standard deviations, however, are affected only little.

All correction terms up to fourth order in $(a / R)$ were included in the calculations. The term with $(a / R)^{3}$ adds about $20-40 \mathrm{keV}$, the term with $(a / R)^{4}$ subtracts about $10-20 \mathrm{keV}$. 
With $f$ and $A E_{\text {corr }}$ assumed to be independent of $A$, the least-squares analysis ${ }^{\dagger}$ of the Coulomb displacement energies of the 42 essentially spherical nuclei resulted in a fit with a standard deviation of about $60 \mathrm{keV}$ only. Moreover, a close inspection of the individual differences between the calculated and experimental energies revealed a surprising correlation with the correction term due to the electromagnetic spinorbit interaction. This correlation is shown in fig. 5 for $f=1.144$ and $\Delta E_{\text {corr }}=+550$ $\mathrm{keV}$. A large positive or negative correction term $\Delta E_{\text {s.o. }}$ results in a large positive or negative deviation of $\Delta E_{\text {calc }}$ from $\Delta E_{\text {exp. }}$. If the term $\Delta E_{\text {s.o. }}$ is artificially set equal to zero, the standard deviation is immediately reduced to about $20 \mathrm{keV}$.

An explanation of this effect is based on the assumption of large amounts of excitations into higher shell-model orbits. It is shown in fig. 2 that by introducing subshell mixing, the line which represents the spin-orbit sum $S_{N}=\sum_{1}^{N} l \cdot \sigma$ is averaged between the two respective neutron numbers. Similarly, by introducing various amounts $p$ of excitations into higher orbits, the $A$-dependence of $S_{N}$ becomes smoother and smoother. Fig. 6 shows the dependence of the averaged sum

$$
\overline{S_{N}}=\sum_{v} \varphi(v, N, \alpha) \boldsymbol{l}_{v} \cdot \sigma_{v},
$$

as a function of $A$. The squares of the occupation numbers are represented by the Fermi function $\varphi(v, N, \alpha)=[1+\exp ((v-N) / \alpha N)]^{-1}$, and the percentage $p$ of excitations into higher orbits is given by

$$
p=\int_{N}^{\infty} \varphi(v, N, \alpha) \mathrm{d} v / \int_{0}^{\infty} \varphi(v, N, \alpha) \mathrm{d} v=\frac{\ln 2}{\ln \left(1+\mathrm{e}^{1 / \alpha}\right)} .
$$

With increasing $p$ the averaged sum $\overline{S_{N}}$ gradually approaches a straight line with a slope of about 0.6 (dotted line).

Least-squares analyses were performed for the 42 nearly semi-magic nuclei and the dependence of the standard deviation $\sigma_{\text {std }}$ on the percentage $p$ of excitations into higher orbits was studied. A significant improvement in the agreement between the experimental and calculated energies was obtained for $p>15 \%$ and there exists a broad minimum in the standard deviation of about $20 \mathrm{keV}$ for $p \approx 30 \%$. It is reassuring to find that a percentage of $p>15 \%$ for excitations into higher shell-model orbits is in good agreement with theoretical estimates ${ }^{28-30}$ ) for the amount of core excitations in the regions of, for example, ${ }^{16} \mathrm{O}$ and ${ }^{40} \mathrm{Ca}$ and ${ }^{208} \mathrm{~Pb}$.

In the least-squares analysis discussed below a term $\Delta E_{\text {s.o. }}$ based on the expression $S_{N}=0.6 N$ has been used for simplicity. Values for $\Delta E_{\text {s.o. }}$. which are obtained this way $(\approx-20 \mathrm{keV}$ approximately independent of $A$ ) differ by less than $\pm 10 \mathrm{keV}$ from those calculated with any $p>15 \%$ but differ by up to $\pm 100 \mathrm{keV}$, of course (see fig. 5),

† It was discovered afterwards that the exchange factor which was used contained a minor numerical mistake. Using the correct cxpression leads to a small decrease in $\Delta E_{\text {eorr }}$ but no change in any of the conclusions. 
from those obtained with $p=0 \%$. Assuming constant values for the ratio $f$ of the excess/core radii and for the correction term $\Delta E_{\text {corr }}$, a standard deviation of $21 \mathrm{keV}$ is obtained for $f=1.144$ and $\Delta E_{\text {corr }}=+550 \mathrm{keV}$. Fig. 7 shows a plot of the individual differences between the experimental and calculated energies as a function of $A$. Only

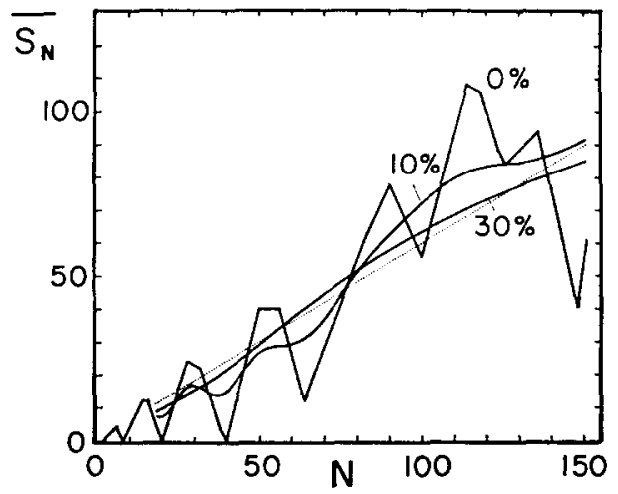

Fig. 6. Plot of the averaged spin-orbit sums $\overline{S_{N}}$ (compare with fig. 2) obtained for percentages of $p=0 \%, 10 \%$ and $30 \%$ excitations into higher orbits. The dotted straight line represents the approximation $\overline{S_{N}}=0.6 \mathrm{~N}$.

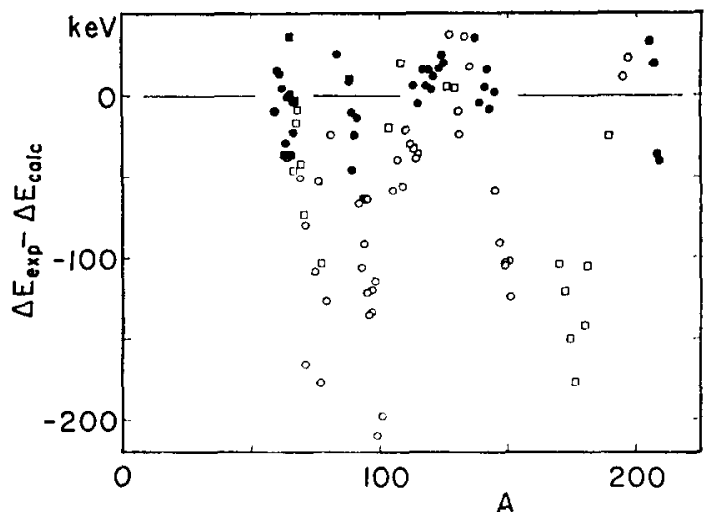

Fig. 7. Plot of the difference between experimental and calculated energies as a function of $A$ for $r_{0}=1.135 \mathrm{fm}, a=0.513 \mathrm{fm}, f=1.144$ and $\Delta E_{\text {corr }}=550 \mathrm{keV}$. Only the filled circles and squares for the nuclei near magic lines were used in the analysis. The energies for all other nuclei are indicated by open circles and squares. Circles and squares represent values with experimental uncertainties less than $30 \mathrm{keV}$ and less than $100 \mathrm{keV}$, respectively. The experimental energies of rotational or vibrational nuclei (nuclei with a static or dynamic deformation in the ground state) are considerably smaller than the energies calculated on the basis of spherical charge distributions.

the filled points for the nuclei near magic proton or neutron numbers were used in the analysis. The remaining open points are clearly lower by up to $200 \mathrm{keV}$ and they show the effect of a decrease in Coulomb energy for rotational $(A \approx 150-190)$ and vibrational $(A \approx 70-80$ and $95-105)$ nuclei. Thus, nuclei with a static or dynamic deforma- 
tion in the ground state have a lower Coulomb energy than corresponding nuclei with spherical charge distributions.

A preliminary comparison between the deformations obtained from the observed reduction in Coulomb displacement energies and the known deformations ${ }^{31,32}$ ) promises excellent agreement. The reduction in Coulomb energy depends in first order on $\beta^{2}$. This quantity is to be interpreted as the square of the deformation parameter $\beta$ for rotational nuclei or the mean-square value $\left\langle\beta^{2}\right\rangle$ for vibrational nuclei (in the

\section{TABLE 2}

Optimized values for the ratio $f \equiv\left\langle r^{2}{ }_{\text {exc }}\right\rangle \frac{1}{2} /\left\langle r^{2}{ }_{\text {core }}\right\rangle \frac{1}{2}=R^{\prime} / R$ and the correction term $\Delta E_{\text {corr }}$ with standard deviations $\sigma_{\text {st.d. }}$.

\begin{tabular}{ccc}
\hline$f$ & $\Delta E_{\text {corr }}(\mathrm{keV})$ & $\sigma_{\text {st. .d. }}(\mathrm{keV})$ \\
\hline 1.12 & $(490-0.93 A)$ & 20.9 \\
1.13 & $(510-0.50 A)$ & 20.8 \\
1.14 & $(540-0.15 A)$ & 21.0 \\
1.144 & $(550)$ & 21.1 \\
1.15 & $(570+0.19 A)$ & 21.4 \\
\hline
\end{tabular}

ground state). The reduction is mostly due to the monopole redistribution of the charge, that is to the increase of the rms radius obtained under the condition of constant density in the nuclear interior. However, more than half of this decrease is compensated by an increase due to the interaction of the multipole part of the potential field with the multipole charge distribution ${ }^{33}$ ). A more detailed investigation of these effects for nuclei with diffuse surfaces and more general shapes will be published elsewhere.

The least-squares analysis for the nearly semi-magic nuclei resulted in a fit with a standard deviation of about $20 \mathrm{keV}$. This result is rather surprising considering the complexity of the correction term $\Delta E_{\text {corr }}$. As mentioned earlier, detailed theoretical calculations for $\Delta E_{\text {corr }}$ have been made for only few nuclei, particularly for the ${ }^{41} \mathrm{Sc}-{ }^{41} \mathrm{Ca}$ isobaric pair. The present results seem to indicate that $\Delta E_{\text {corr }}$ must depend relatively smoothly on $A$, at least for nuclei which are not too light.

In the preceding analysis the quantities $f$ and $\Delta E_{\text {corr }}$ were assumed to be constant. Another choice is to assume a linear $A$-dependence for $\Delta E_{\text {corr }}$ for any tixed value of $f$. Table 2 shows the results [see also eq. (46)]. The standard deviation $\sigma_{\text {st.d. }}$ exhibits a broad minimum near $f=1.125$. If therefore appears that the most reasonable values for $f$ lie between $f=1.10$ and $f=1.15$. The required values for $\Delta E_{\text {corr }}$ lie between $450 \pm 100 \mathrm{keV}$ in the light nuclei and $350 \pm 250 \mathrm{keV}$ in the heavy nuclei. The above range for $f$ also leads to very reasonable values for the neutron halo as will be shown in sect. 8. More definite statements about $f$ cannot be made due to the strong correlations between $f$ and $\Delta E_{\text {oorr }}$ and due to the limited knowledge about $\Delta E_{\text {corr }}$ particularly its $A$-dependence. 


\section{The neutron halo}

If the ratio $f \equiv\left\langle r_{\text {exc }}^{2}\right\rangle^{\frac{1}{2}} /\left\langle r_{\text {core }}^{2}\right\rangle^{\frac{1}{2}}$ for the radius of the neutron excess to the proton core is known as well as the radius of the proton core, it is possible to calculate for any nucleus the radius of the neutron excess in the ground state, the radius of the excess proton in the analogue state, the radius of all neutrons in the ground state and the radius of all protons in the analogue state. The following expressions are obtained for the rms radii:

(i) all protons in ground state: $\left\langle r_{\text {core }}^{2}\right\rangle^{\frac{1}{2}}$,

(ii) neutron excess in ground state: $f\left\langle r_{\text {core }}^{2}\right\rangle^{\frac{1}{2}}$,

(iii) excess proton in analogue state: $f\left\langle r_{\text {core }}^{2}\right\rangle^{\frac{1}{2}}$,

(iv) all neutrons in ground state: $\sqrt{\left(Z+(N-Z) f^{2}\right) / N}\left\langle r_{\text {core }}^{2}\right\rangle^{\frac{1}{2}}$,

(v) all protons in analogue state: $\sqrt{\left(Z+f^{2}\right) /(Z+1)}\left\langle r_{\text {core }}^{2}\right)^{\frac{1}{2}}$.

If, for example, we assume a radius for the neutron excess in ${ }^{208} \mathrm{~Pb}$ which is $12.5 \%$ larger than the radius of the core, the radius of all neutrons in the ground state is $4.7 \%$ larger and that of all protons in the analogue state is $0.2 \%$ larger than that of the core. Using eq. (54) the neutron halo $H$ in the ground state of any nucleus can be obtained from the expression

$$
H \equiv\left\langle r_{\mathrm{n}}^{2}\right\rangle^{\frac{1}{2}}-\left\langle r_{\mathrm{p}}^{2}\right\rangle^{\frac{1}{2}}=\left\{\sqrt{\frac{Z+(N-Z) f^{2}}{N}}-1\right\} \sqrt{\frac{3}{5}} R,
$$

where $R$ is the equivalent charge radius. It can be taken from experiment or can otherwise be calculated from eq. (6) with $r_{v}=1.135 \mathrm{fm}$ and $a-0.513 \mathrm{fm}$. Fig. 8 shows a plot of $H$ as a function of $A$ for the nuclei along the stability line which is approximately characterized by ${ }^{34}$ )

$$
\widetilde{T}_{\mathrm{Z}}=\frac{0.2 A^{2}}{200+A}
$$

For the ratio $f$ the values $1.10,1.125$ and 1.5 were used. As was shown in sect. 7 , these values cover the range which is most reasonable. The thin line in fig. 8 has been calculated by Myers and Swiatecki ${ }^{35,36}$ ) on the basis of the droplet model, a refinement of the liquid-drop model. (The same eq. (57) has been used here to describe the stability line.) Excellent agreement exists, and the curves from the droplet model and from the present work with $f=1.125$ practically coincide over the entire range. A comparison between the two expressions for $H$ shows that $f$ is closely related to the symmetry energy and the effective surface stifness.

Table 3 gives values for the neutron halo $H$ obtained from a variety of procedures, and for a selected group of nuclei. The table has essentially been reproduced from the publication of Lee and Cusson ${ }^{37}$ ) except for the present results. Good agreement 


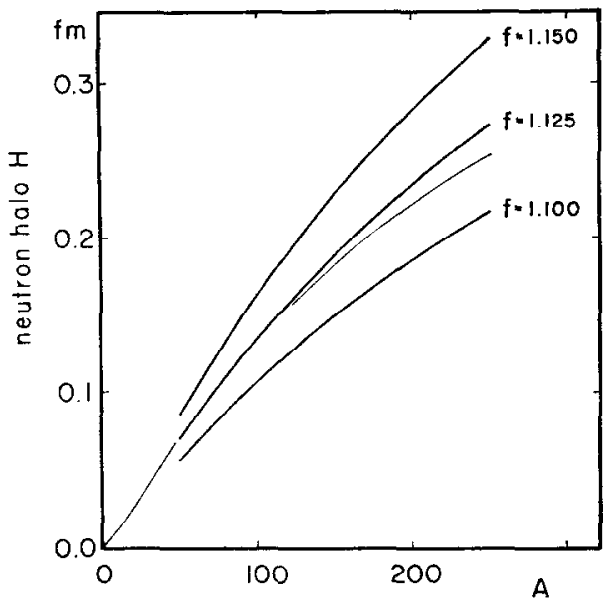

Fig. 8. Neutron halo $H \equiv\left\langle r_{\mathrm{n}}{ }^{2}\right\rangle \frac{1}{2}-\left\langle r_{\mathrm{p}}{ }^{2}\right\rangle \frac{1}{2}$ for nuclei along the stability line as a function of $A$. The three heavy lines are for $f=1.10,1.125$ and 1.15. The thin line is derived from the droplet model of Myers and Swiatecki ${ }^{33,36}$ ).

TABLE 3

Neutron halo $H \equiv\left\langle r_{\mathrm{n}}^{2}\right\rangle \frac{1}{2}-\left\langle r_{\mathrm{p}}{ }^{2}\right\rangle \frac{1}{2}$ in units of $\mathrm{fm}$

\begin{tabular}{|c|c|c|c|c|c|c|c|c|}
\hline Nucleus & $\begin{array}{l}\text { Hartree- } \\
\text { Fock }\end{array}$ & $\begin{array}{l}\text { Droplet } \\
\text { model d) }\end{array}$ & $\begin{array}{c}\text { Coulomb } \\
\text { dis- } \\
\text { placement } \\
\text { energies } \\
\text { this work }\end{array}$ & $\begin{array}{l}\text { Coulomb } \\
\text { dis- } \\
\text { placement } \\
\text { enzrgies } \\
\text { other work }\end{array}$ & $\begin{array}{l}\text { Statis- } \\
\text { tical } \\
\text { theory }\end{array}$ & $\begin{array}{l}\text { Woods- } \\
\text { Saxon } \\
\text { poten- } \\
\text { tials }{ }^{\circ} \text { ) }\end{array}$ & $\begin{array}{c}\text { Single- } \\
\text { particle } \\
\text { poten- } \\
\text { tials }\end{array}$ & $\begin{array}{c}\text { Proton } \\
\text { elastic } \\
\text { scattering }{ }^{\mathbf{k}} \text { ) }\end{array}$ \\
\hline${ }^{48} \mathrm{Ca}$ & $\begin{array}{l}0.24^{a} \text { ) } \\
\left.0.23^{b}\right) \\
\left.0.137^{c}\right)\end{array}$ & 0.160 & $(0.13 \pm 0.03)$ & $\left.0.06^{\mathrm{c}}\right)$ & 0.10 & 0.25 & $\left.0.45^{1}\right)$ & \\
\hline${ }^{56} \mathrm{Fe}$ & $\left.0.074^{c}\right)$ & 0.062 & $(0.07 \pm 0.01)$ & & & & $0.24^{1}$ ) & $-0.04 \pm 0.15$ \\
\hline${ }^{58} \mathrm{Ni}$ & $\left.0.020^{c}\right)$ & 0.023 & $0.03 \pm 0.01$ & & & & $0.14^{1}$ ) & $0.01 \pm 0.18$ \\
\hline${ }^{59} \mathrm{Co}$ & $\left.0.088^{c}\right)$ & 0.077 & $(0.08 \pm 0.02)$ & & & & & $-0.03 \pm 0.16$ \\
\hline${ }^{60} \mathrm{Ni}$ & $0.065^{c}$ ) & 0.057 & $0.06 \pm 0.01$ & & & & $\left.0.23^{i}\right)$ & $0.03 \pm 0.16$ \\
\hline${ }^{62} \mathrm{Ni}$ & $\left.0.114^{c}\right)$ & 0.090 & $0.09 \pm 0.02$ & $\left.0.04^{\mathrm{e}}\right)$ & & 0.12 & & \\
\hline${ }^{90} \mathrm{Zr}$ & $\begin{array}{l}\left.0.13^{a}\right) \\
\left.0.12^{b}\right) \\
\left.0.150^{c}\right)\end{array}$ & 0.108 & $0.11 \pm 0.02$ & $\left.0.02^{5}\right)$ & & & & \\
\hline${ }^{120} \mathrm{Sn}$ & $\left.0.246^{\mathrm{c}}\right)$ & 0.175 & $0.17 \pm 0.04$ & $\begin{array}{l}\left.0.05^{1}\right) \\
\left.0.07^{\circ}\right)\end{array}$ & & 0.25 & $\left.0.44^{i}\right)$ & $0.15 \pm 0.19$ \\
\hline${ }^{208} \mathrm{~Pb}$ & $\begin{array}{l}\left.0.29^{a}\right) \\
\left.0.23^{b}\right) \\
\left.0.25^{c}\right)\end{array}$ & 0.237 & $0.25 \pm 0.05$ & $\begin{array}{l}\left.0.09^{f}\right) \\
\left.0.15^{e}\right) \\
\left.0.08^{8}\right)\end{array}$ & 0.11 & 0.28 & $\begin{array}{l}\left.0.64^{j}\right) \\
\left.0.62^{i}\right)\end{array}$ & $0.13 \pm 0.25$ \\
\hline
\end{tabular}

The table is essentially taken from Lee and Cusson ${ }^{37}$ ). The values from this work given in parenthesis are for $Z<28$.

Bethe and Siemens ${ }^{38}$ ) calculated a value of $-0.02 \mathrm{fm}$ for the difference of the half-density radii in ${ }^{208} \mathrm{~Pb}$.
a) Ref. ${ }^{39}$ ).
b) Ref. ${ }^{40}$ ),
c) Ref. ${ }^{37}$ ).
d) Ref. ${ }^{35}$ ).
c) Ref. ${ }^{1}$ ).
f) Ref. ${ }^{4}$ ).
g) Ref. $\left.{ }^{5}\right)\left({ }^{209} \mathrm{~Pb}\right)$.
h) Ref. ${ }^{41}$ ).
1) Ref. ${ }^{42}$ ).
J) Ref. ${ }^{43}$ ).
k) Ref. ${ }^{44}$ ). 
exists among the results obtained by three completely different procedures, namely those obtained from Hartree-Fock calculations, from the droplet model and from the present work using Coulomb displacement energies. Also, the values obtained by Nolen and Schiffer ${ }^{1}$ ) based on wave functions for the core particles and the excess neutrons generated in Woods-Saxon potential wells agree quite well. The values obtained earlier from Coulomb displacement energies are probably too small. The fact that there exists such good agreement between the results from four different methods, despite their inherent shortcomings, lends support to the suggestion that the results are indeed close to the true values for the neutron halo.

\section{Coulomb energies and atomic masses}

Coulomb energies contribute significantly to the atomic masses, and an accurate determination of this contribution is desirable. Based on the conclusions of the preceding sections we will give here an analytic expression which can be used in connection with liquid-drop-type mass equations. The electromagnetic energy can be expressed as

$$
E=E_{\mathrm{dir}}+E_{\mathrm{exch}}+E_{\mathrm{s.o} .} .
$$

A correction term $E_{\text {corr }}$ related to the earlier $\Delta E_{\text {corr }}$ will be neglected. The quantity $E_{\text {dir }}$ represents the direct Coulomb energy, $E_{\text {exch }}$ the exchange contributions and $E_{\text {s.o. }}$ the electromagnetic spin-orbit contribution. We have

$$
\begin{aligned}
E_{\mathrm{dir}} & =\frac{3}{5} \frac{Z(Z-1) e^{2}}{R} C_{\mathrm{dir}}, \\
E_{\mathrm{exch}} & =-3\left(\frac{3}{16 \pi}\right)^{\frac{3}{3}} \frac{e^{2} Z^{4 / 3}}{R} C_{\mathrm{exch}}+\frac{3}{5} \frac{Z e^{2}}{R} C_{\mathrm{dir}}, \\
E_{\mathrm{s} . \mathrm{o} .} & =-\mu_{0}^{2}\left\{\left(g_{\mathrm{p}}-1\right) \sum_{1}^{Z} l \cdot \sigma+g_{\mathrm{n}} \sum_{1}^{N} l \cdot \sigma\right\} \frac{Z}{R^{3}} C_{\text {s.o. }},
\end{aligned}
$$

where $R$ is again the equivalent charge radius which can be either taken from experiment or be calculated from

$$
R=r_{0} A^{3}\left\{1+\frac{5}{6} \pi^{2}\left(\frac{a}{r_{0} A^{\frac{3}{3}}}\right)^{2}-\frac{7}{24} \pi^{4}\left(\frac{a}{r_{0} A^{5}}\right)^{4}\right\},
$$

with $r_{0}=1.135 \mathrm{fm}$ and $a=0.513 \mathrm{fm}$. The diffuseness correction terms are

$$
\begin{aligned}
& C_{\text {dir }}=1+18.030\left(\frac{a}{R}\right)^{3}-\frac{7}{8} \pi^{4}\left(\frac{a}{R}\right)^{4}, \\
& C_{\text {exch }}=1-1.336\left(\begin{array}{l}
a \\
R
\end{array}\right)+7.127\left(\frac{a}{R}\right)^{2}-18.210\left(\frac{a}{R}\right)^{3}+83.406\left(\frac{a}{R}\right)^{4}, \\
& C_{\text {s.o. }}=1+\pi^{2}\left(\frac{a}{R}\right)^{2}-\frac{7}{8} \pi^{4}\left(\frac{a}{R}\right)^{4} .
\end{aligned}
$$


The last expression is obtained from eqs. (5), (44) and (45) with $f=1$. The spin-orbit sums $\sum_{1}^{Z} \boldsymbol{l} \cdot \boldsymbol{\sigma}$ and $\sum_{1}^{N} \boldsymbol{l} \cdot \boldsymbol{\sigma}$ can be approximated by $0.6 Z$ and $0.6 \mathrm{~N}$, respectively. Also, $e^{2}=1.440 \mathrm{MeV} \cdot \mathrm{fm}, \mu_{0}^{2}=15.9 \mathrm{keV} \cdot \mathrm{fm}^{3}, g_{\mathrm{p}}-1=4.570$ and $g_{\mathrm{n}}=-3.870$.

Contributions from the cubic and quartic terms in $C_{\text {exch }}$ are small and can generally be neglected. Since $g_{\mathrm{p}}-1$ and $g_{\mathrm{n}}$ in the expression for the electromagnetic spin-orbit interaction have opposite sign, the contributions from the protons and the neutrons cancel to a large extent. Therefore, the term $E_{\text {s.o. }}$ is generally quite small and can often be neglected. This result is contrary to the situation for the Coulomb displacement energies where the difference of two terms of opposite sign has to be considered for the electromagnetic spin-orbit term.

Eq. (58) is valid for spherical nuclei. If static or dynamic deformations of the nuclear shape are considered, an approximate correction factor $\left(1-\beta^{2} / 4 \pi\right)$ must be applied to the direct Coulomb energy term ${ }^{33}$ ). A more detailed description of the Coulomb energies of rotational and vibrational nuclei with diffuse surfaces and more general shapes will be published at a later time.

\section{Summary}

A phenomenological Coulomb energy equation has been used in a least-squares analysis of nuclei which are presumably nearly spherical in shape. The parameters for the ground state charge distributions have been chosen to obtain agreement with the results of electron scattering and muon capture X-ray experiments ${ }^{17}$ ). Using the ratio $f$ of the rms radii of the neutron excess to that of the proton core and the correction term $\Delta E_{\text {corr }}$ as adjustable parameters, a standard deviation of $\sigma_{\text {st.d. }}=21$ $\mathrm{keV}$ has been obtained for values of $f$ in the range 1.10 to 1.15 and $\Delta E_{\text {corr }}$ ranging with appreciable uncertainties from about +450 in the light nuclei to about $+350 \mathrm{keV}$ in the heavy nuclei. However, the good agreement is obtained only if large amounts of at least $15 \%$ of excitations into higher shell-model orbits are assumed for all nuclei, an assumption which strongly affects the contributions from the electromagnetic spin-orbit interaction.

The experimental Coulomb displacement energies of nuclei which lie off the magic lines are smaller by up to $200 \mathrm{keV}$ than corresponding spherical nuclei. They clearly exhibit the expected decrease in Coulomb energy for vibrational and rotational nuclei.

Neutron halos have been calculated using a ratio $f \equiv\left\langle r_{\text {exc }}^{2}\right\rangle^{\frac{1}{2}} /\left\langle r_{\text {core }}^{2}\right\rangle^{\frac{1}{2}}=1.125 \pm$ 0.125 for the radii of the neutron excess and the proton core. The results are found in excellent agreement with the results of other calculations (see fig. 8 and table 3).

Thanks are due to Dr. J. P. Draayer for helpful discussions and the careful reading of the manuscript. 


\section{Appendix A}

FERMI INTEGRALS OF INTEGER ORDER

The Fermi function

$$
f(\xi-k)=\{1+\exp (\xi-k)\}^{-1},
$$

arises in the treatment of a variety of theoretical problems. Frequently Fermi integrals $F_{n}(k, x)$ defined by

$$
F_{n}(k, x)=\int_{0}^{x} \xi^{n} f(\xi-k) \mathrm{d} \xi,
$$

have to be evaluated. For integer $n \geqq 0$ and in the limit $x \rightarrow \infty$ the integrals have been considered in detail by Rhodes $\left.{ }^{18}\right)$. The results for $k>0$ are

and for $n>0$

$$
F_{0}(k, \infty)=\ln \left(1+\mathrm{e}^{k}\right),
$$

$$
F_{n}(k, \infty)=S_{n}(k)+(-1)^{n} F_{n}(-k, \infty),
$$

where $S_{n}(k)$ is a polynomial of order $n+1$ in $k$ given by

$$
\begin{aligned}
S_{n}(k) & =\sum_{m=0}^{\leqq \frac{1}{2}(n+1)} \frac{2 n !}{(n-2 m+1) !}\left(1-\frac{1}{2^{2 m-1}}\right) \zeta(2 m) k^{n-2 m+1} \\
& =\frac{1}{n+1} k^{n+1}+\sum_{m=1}^{\leqq \frac{1}{2}(n+1)} \frac{2 n !}{(n-2 m+1) !}\left(1-\frac{1}{2^{2 m-1}}\right) \zeta(2 m) k^{n-2 m+1} .
\end{aligned}
$$

Here, $\zeta(2 m)$ is the Riemann $\zeta$-function which can simply be expressed in terms of powers of $\pi$ and the Bernouilli numbers ${ }^{19}$ ). The Fermi integral for negative arguments can be represented by the infinite sum

$$
F_{n}(-k, \infty)=n ! \sum_{l=1}^{\infty}(-1)^{l+1} \frac{\mathrm{e}^{l k}}{l^{n+1}} .
$$

For sufficiently large $k$ the value of this function is small and $F_{n}(k, \infty)$ can be approximated by the polynomial $S_{n}(k)$. If $k$ is not large, the correction term $(-1)^{n} F_{n}$ $(-k, \infty)$ must be included. But even in this case just one or at most a few terms of eq. (A.6) need be considered. Numerical values for $(1 / n !) F_{n}(-k, \infty)$ and $0 \leqq k \leqq 4$ have been tabulated by Rhodes ${ }^{19}$ ).

Another important relationship given by Rhodes ${ }^{18}$ ) is

$$
\frac{\mathrm{d}}{\mathrm{d} k} F_{n}(k, \infty)=n F_{n-1}(k, \infty),
$$

for all $n>1$.

The integral $F_{n}(k, x)$ for finite values of $x$ and $n=0$ can be evaluated immediately. The result is

$$
F_{0}(k, x)=\ln \left(\frac{1+\exp (k)}{1+\exp (k-x)}\right) .
$$


For integer $n \geqq 1$ the integrals $F_{n}(k, x)$ can be calculated by introducing the expansions

$$
f(\xi-k)= \begin{cases}\sum_{l=0}^{\infty}(-1)^{l} \mathrm{e}^{l(\xi-k)} & \text { for } 0 \leqq \xi<k, \\ \sum_{l=0}^{\infty}(-1)^{l} \mathrm{e}^{(l+1)(k-\xi)} & \text { for } k<\xi .\end{cases}
$$

The resulting series can be integrated term by term since they are uniformly convergent. Integrating by parts, one obtains the general expressions

$$
F_{n}(k, x)=\left\{\begin{array}{cc}
\frac{1}{n+1} x^{n+1}+\sum_{m=0}^{n} \frac{(-1)^{m} n !}{(n-m) !} x^{n-m} \sum_{l=1}^{\infty} \frac{(-1)^{l}}{l^{m+1}} \mathrm{e}^{l(x-k)}+(-1)^{n} F_{n}(-k, \infty) \\
\text { for } 0 \leqq x<k, \\
S_{n}(k)+\sum_{m=0}^{n} \frac{n !}{(n-m) !} x^{n-m} \sum_{l=1}^{\infty} \frac{(-1)^{l}}{l^{m+1}} \mathrm{e}^{l(k-x)}+(-1)^{n} F_{n}(-k, \infty) \\
\text { for } k<x .
\end{array}\right.
$$

In the limit $x \rightarrow \infty$ the second term in the second equation disappears and eq. (A.4) is reproduced. For $x \rightarrow k$ the expressions (A.10) are not absolutely convergent. One obtains

$$
\begin{aligned}
F_{n}(k, k)=\frac{1}{n+1} k^{n+1}-(\ln 2) k^{n}+\sum_{m=1}^{n} \frac{(-1)^{m+1} n !}{(n-m) !}\left(1-\frac{1}{2^{m}}\right) \zeta(m+1) k^{n-m} \\
+(-1)^{n} F_{n}(-k, \infty) .
\end{aligned}
$$

The Fermi integral $F_{2}(k, x)$ is widely used in the present work. By specializing eqs. (A.10) and (A.11) one obtains

$$
\begin{gathered}
F_{2}(k, x)=\left\{\begin{array}{c}
\frac{1}{3} x^{3}+\sum_{l=1}^{\infty}(-1)^{l}\left(\frac{x^{2}}{l}-\frac{2 x}{l^{2}}+\frac{2}{l^{3}}\right) \mathrm{e}^{l(x-k)}+2 \sum_{l=1}^{\infty}(-1)^{l+1} \frac{\mathrm{e}^{-l k}}{l^{3}} \\
\text { for } 0 \leqq x<k, \\
\frac{1}{3} k^{3}+\frac{1}{3} \pi^{2} k+\sum_{l=1}^{\infty}(-1)^{l}\left(\frac{x^{2}}{l}+\frac{2 x}{l^{2}}+\frac{2}{l^{3}}\right) \mathrm{e}^{l(k-x)}+2 \sum_{l=1}^{\infty}(-1)^{l+1} \frac{\mathrm{e}^{-l k}}{l^{3}} \\
\text { for } k<x,
\end{array}\right. \\
F_{2}(k, k)=\frac{1}{3} k^{3}-(\ln 2) k^{2}+\frac{1}{6} \pi^{2} k-\frac{3}{2} \zeta(3)+2 \sum_{l=1}^{\infty}(-1)^{l+1} \frac{\mathrm{e}^{-l k}}{l^{3}}
\end{gathered}
$$

For some applications it is practical to express the Fermi function $f(\xi-k)$ in terms of a step function $S(\xi-k)$ and derivative of the $\delta$-function [see for example ref. $\left.{ }^{19}\right)$ ]

$$
f(\xi-k) \approx S(\xi-k)-2 \sum_{l=1}^{\infty}\left(1-\frac{1}{2^{2 l-1}}\right) \zeta(2 l) \delta^{(2 l-1)}(\xi-k) .
$$


In this approximation one obtains

$$
F_{n}(k, x) \approx \begin{cases}\frac{1}{n+1} x^{n+1} & \text { for } 0 \leqq x<k, \\ S_{n}(k) & \text { for } k<x,\end{cases}
$$

which is a good representation only for $x / k \ll 1$ and $x / k \gg 1$, respectively. A graphical display of the function $\left(1 / x^{2}\right) F_{2}(k, x) / F_{2}(k, \infty)$ based on eqs. (A.10) and (A.15) is shown in fig. 1 .

\section{Appendix B}

NUMERICAL EVALUATION OF INTEGRALS RELATED TO THE FERMI FUNCTION

(i) The integral $\int_{0}^{\infty}\left(\left[F_{2}(k, \xi)\right]^{2} / \xi^{2}\right) \mathrm{d} \xi$.

The above integral with $k=c / a$ is that of eq. (13). It can be evaluated by using the expansions for $F_{2}(k, x)$ given in eq. (A.12). The correction term $F_{2}(-k, \infty)$ will be neglected throughout. Integrating from 0 to $k$ to infinity, one has to evaluate integrals of the type $\int \xi^{m} \mathrm{e}^{ \pm l(\xi-k)} \mathrm{d} \xi$. They can be integrated by parts. Ordering the results in decreasing powers of $k$ gives

$$
\int_{0}^{\infty} \frac{\left[F_{2}(k, \xi)\right]^{2}}{\xi^{2}} \mathrm{~d} \xi=\frac{2}{15}\left(k^{5}+\frac{5}{6} \pi^{2} k^{3}+b_{1} k^{2}-\frac{1}{6} \pi^{2} k\right) .
$$

Eq. (15) for $E_{\text {dir }}$ is finally obtained from eq. (13) by substituting (i) the above result for the integral, (ii) $S_{2}(k)=\frac{1}{3} k^{3}\left(1+\pi^{2}(1 / k)^{2}\right)$ for $F_{2}(k, \infty)$, and (iii) the radius $c$ by $R$ according to eq. (5). The coefficients $b_{1}$ and $b_{2}$ in eq. (16) are

with

$$
\begin{gathered}
b_{1}=15\left[\frac{3}{4} \zeta(3)+S_{0}\right], \\
b_{2}=-\frac{7}{8} \pi^{4},
\end{gathered}
$$

$$
S_{0}=\sum_{i=1}^{\infty} \sum_{j=1}^{\infty} \frac{(-1)^{i+j}}{i j(i+j)} \text {. }
$$

The coefficient $b_{1}$ has also been derived by Lindner ${ }^{20}$ ). The sum $S_{0}$ converges rapidly in the form ${ }^{45}$ )

$$
S_{0}=\frac{1}{4} \pi^{2} \ln 2+\sum_{l=1}^{\infty} \frac{1}{l^{2}} \sum_{m=1}^{l} \frac{(-1)^{m}}{m} .
$$

(ii) The integral $\int_{0}^{\infty}\left(F_{2}(k, \xi) F_{2}\left(k^{\prime}, \xi\right) / \xi^{2}\right) \mathrm{d} \xi$.

The above integral with $k=c / a$ and $k^{\prime}=c^{\prime} / a$ is required for an evaluation of cq. (19). It is more difficult to handle. Again, the aforementioned expansions for $F_{2}(k, \xi)$ and $F_{2}\left(k^{\prime}, \xi\right)$ can be used and the integral calculated over the regions from zero to $k$ to $k^{\prime}=h k$ to infinity. The procedure is identical to that mentioned in (i) but unlike 
that case the coefficients for the terms in decreasing powers of $k$ are functions of $k$ and not constant. These coefficients include the functions

$$
\zeta(n, x) \equiv \sum_{l=1}^{\infty} \frac{\mathrm{e}^{-l x}}{l^{n}}
$$

as well as other functions defined by infinite double sums related, for example, to that given in eq. (B.4). It can be shown, however, that by properly rearranging these sums, the rather complicated expressions can be greatly simplified. The final result is

$$
\int_{0}^{\infty} \frac{F_{2}(k, \xi) F_{2}\left(k^{\prime}, \xi\right)}{\xi^{2}} \mathrm{~d} \xi=\frac{2}{15}\left\{b_{3}(h) k^{5}+b_{4}(h, k) k^{3}+b_{5}(h, k) k^{2}+b_{6}(h, k) k\right\},
$$

where $b_{3}$ to $b_{6}$ are defined by eqs. (21)-(24).

The functions $\zeta(n, x)$ have simple limits for $x \rightarrow 0$ and $x \rightarrow \infty$. We have $\zeta(n, 0)=$ $\zeta(n)$ and $\zeta(n, \infty)=0$, where $\zeta(n)$ is again the Riemann $\zeta$-function. For finite values of $x$ the functions can be approximated to any desired accuracy by finite sums.

Eq. (20) for $\Delta E_{\text {dir }}$ is obtained by substituting the above integral and the appropriate expressions for the Fermi integrals into eq. (19). The half-density radius $c$ and the ratio $h=c^{\prime} / c$ have not been replaced explicitly.

(iii) The integral $\int_{0}^{\infty} \xi^{2}[f(\xi-k)]^{*} \mathrm{~d} \xi$.

The above integral with $k=c / a$ is from eq. (35). It can be evaluated in a way very similar to the integral $F_{2}(k, x)$, if instead of the power series expansion (A.9) for $f(\xi-k)$ one uses the expansions

$$
[f(\xi-k)]^{4 / 3}= \begin{cases}\sum_{l=0}^{\infty}\left(\begin{array}{c}
-\frac{4}{3} \\
l
\end{array}\right) \mathrm{e}^{l(\xi-k)} & \text { for } 0 \leqq \xi<k, \\
\sum_{l=0}^{\infty}\left(\begin{array}{c}
-\frac{4}{3} \\
l
\end{array}\right) \mathrm{e}^{(l+4 / 3)(k-\xi)} & \text { for } k<\xi .\end{cases}
$$

Again, integrating by parts is required. Ordering in decreasing powers of $k$ and replacing $c$ by $R$ one obtains eq. (36) with the correction term $C_{\text {exch }}$ of eq. (37). The coefficients are

where

$$
\begin{aligned}
b_{7} & =\frac{9}{4}+3 S_{1}, \\
b_{8} & =\frac{27}{8}-\frac{1}{6} \pi^{2}-6 S_{2}, \\
b_{9} & =\frac{81}{32}+\frac{9}{4} \pi^{2}+3 \pi^{2} S_{1}+6 S_{3}, \\
b_{10} & =\frac{117}{16} \pi^{2}-\frac{77}{72} \pi^{4}-13 \pi^{2} S_{2},
\end{aligned}
$$

$$
\begin{aligned}
& S_{1}=\sum_{l=1}^{\infty}\left(\begin{array}{c}
-\frac{4}{3} \\
l
\end{array}\right)\left(\frac{1}{l}+\frac{1}{l+\frac{4}{3}}\right), \\
& S_{2}=\sum_{l=1}^{\infty}\left(\begin{array}{c}
-\frac{4}{3} \\
l
\end{array}\right)\left(\frac{1}{l^{2}}-\frac{1}{\left(l+\frac{4}{3}\right)^{2}}\right),
\end{aligned}
$$




$$
S_{3}=\sum_{l=1}^{\infty}\left(\begin{array}{c}
-\frac{4}{3} \\
l
\end{array}\right)\left(\frac{1}{l^{3}}+\frac{1}{\left(l+\frac{4}{3}\right)^{3}}\right) .
$$

The expression for $b_{7}$ and its numerical value have been derived by Lindner ${ }^{20}$ ). The sums $S_{2}$ and $S_{3}$ converge rapidly. The sum $S_{1}$, however, is not even absolutely convergent and a computer calculation is not practical. However, its value can be calculated to any desired accuracy by recognizing that the integral from zero to one of the sum

$$
\sum_{l=1}^{\infty}\left(\begin{array}{c}
-\frac{4}{3} \\
l
\end{array}\right) x^{l+\alpha}=\frac{x^{x}}{(1+x)^{4 / 3}}-x^{x}
$$

reproduces $S_{1}$. Thus, $S_{1}$ can bə written as

$$
S_{1}=\int_{0}^{1}\left\{\frac{1}{x(1+x)^{4 / 3}}-\frac{1}{x}+\frac{x^{\frac{1}{3}}}{(1+x)^{4 / 3}}-x^{\frac{3}{3}}\right\} \mathrm{d} x
$$

which is easily reduced to elementary functions.

(iv) The integral $\int_{0}^{\infty}(1 / \xi) f\left(\xi-k^{\prime}\right) F_{2}(k, \xi) \mathrm{d} \xi$.

The above integral with $k^{\prime}=h c / a$ and $k=c / a$ is from eq. (43). It is easy to evaluate in the $\delta$-function approximation with $F_{2}(k, \xi)$ given by eq. (14). Using eq. (A.14) to represent $f\left(\xi-k^{\prime}\right)$ one obtains integrals including integrals of the type

$$
\int_{0}^{\infty} g(\xi) \delta^{(n)}\left(\xi-\xi_{0}\right) \mathrm{d} \xi=(-1)^{n} g^{(n)}\left(\xi_{0}\right)
$$

which can immediately be solved. The final result for the electromagnetic spin-orbit term is given by eqs. (44) and (45).

\section{References}

1) J. A. Nolen and J. P. Schiffer, Ann. Rev. Nucl. Sci. 19 (1969) 471

2) J. A. Nolen and J. P. Schiffer, Phys. Lett. 29B (1969) 396

3) J. P. Schiffer, J. A. Nolen and N. Williams, Phys. Lett. 29B (1969) 399

4) E. Friedman and B. Mandelbaum, Nucl. Phys. A135 (1969) 472

5) N. Auerbach, J. Hüfner, A. K. Kerman and C. M. Shakin, Phys. Rev. Lett. 23 (1969) 484

6) E. H. Auerbach, S. Kahana and J. Weneser, Phys. Rev. Lett. 23 (1969) 1253

7) E. H. Auerbach, S. Kahana, C. K. Scott and J. Weneser, Phys. Rev. 188 (1969) 1747

8) C. W. Wong, Nucl. Phys. A151 (1970) 323

9) J. Damgaard, C. K. Scott and E. Osnes, Nucl. Phys. A154 (1970) 12

10) J. W. Negele, Nucl. Phys. A165 (1971) 305

11) Nguyen van Giai, D. Vautherin, M. Veneroni and D. M. Brink, to be published

12) J. Jänecke, Isospin in nuclear physics, ed. D. H. Wilkinson (North-Holland, Amsterdam, 1968) ch. 8

13) B. C. Carlson and I. Talmi, Phys. Rev. 96 (1954) 436

14) K. T. Hecht, Nucl. Phys. A102 (1967) 11; A114 (1968) 280

15) L. R. B. Elton, Nucl. Phys. 5 (1958) 173; 8 (1958) 396

16) U. Meyer-Berkhout et al, Ann. of Phys. 8 (1959) 119 
17) L. R. B. Elton, Nuclear radii, Landolt-Börnstein, new series vol. I/4 (Springer, Berlin, Heidelberg, New York, 1967) p. 1;

R. Hofstadter and H. R. Collard, ibid., p. 21

18) P. Rhodes, Proc. Roy. Soc. A204 (1950) 396

19) A. H. Wilson, The theory of metals (Cambridge, University Press, 1958) p. 330

20) A. Lindner, Z. Phys. 211 (1968) 195; 219 (1969) 1

21) H. A. Bethe and R. F. Bacher, Rev. Mod. Phys. 8 (1936) 82

22) H. A. Bethe, Phys. Rev. 54 (1938) 436

23) S. Sengupta, Nucl. Phys. 21 (1960) 542

24) D. R. Inglis, Phys. Rev. 82 (1951) 181

25) R. H. Fulmer and W. W. Daehnick, Phys. Rev. 139 (1965) B579

26) B. F. Bayman and N. M. Hintz, Phys. Rev. 172 (1968) 1113

27) J. Jänecke, Nucl. Phys. A114 (1968) 433

28) G. E. Brown and A. M. Green, Nucl. Phys. 75 (1966) 401;

E. Rost, Phys. Lett. 21 (1966) 87

29) W. J. Gerace and A. M. Green, Nucl. Phys. A93 (1967) 110

30) D. Agassi, V. Gillet and A. Lumbroso, Nucl. Phys. A130 (1969) 129;

J. V. Vergados, Phys. Lett. 34B (1971) 458

31) K. E. G. Löbner, M. Vetter and V. Hönig, Nucl. Data Tables A7 (1970) 495

32) P. H. Stelson and L. Grodzins, Nucl. Data Tables A1 (1965) 21

33) L. Wilets, Theories of nuclear fission (Oxford Clarendon Press, 1964)

34) A. E. S. Green, Nuclear physics (McGraw Hill, New York, 1955) p. 250

35) W. D. Myers, Phys. Lett. 30B (1969) 451

36) W. D. Myers and W. J. Swiatecki, Ann. of Phys. 55 (1969) 395

37) H. C. Lee and R. Y. Cusson, Phys. Lett., to be published

38) H. A. Bethe and P. J. Siemens, Phys. Lett. 27B (1968) 549

39) R. M. Tarbutton and K. T. R. Davies, Nucl. Phys. A120 (1968) 1

40) D. Vautherin and M. Veneroni, Phys. Lett. 29B (1969) 203

$41)$ D. A. Brueckner, J. R. Buchler, R. C. Clark and R. J. Lombard, Phys. Rev. 181 (1969) 1543

42) C. J. Batty and G. W. Greenlees, Nucl. Phys. A133 (1969) 673

43) L. R. B. Elton, Phys. Lett. B26 (1968) 689

44) G. W. Greenlees, W. Makofske and G. J. Pyle, Phys. Rev. C1 (1970) 1145

45) A. Lindner, private communication 